\title{
Selbstkompensierende Kapazitätsmessung mittels eines einfachen OPVs für eine universelle Sensorschnittstelle
}

\author{
Robert Freier, Andreas König \\ Lehrstuhl für Integrierte Sensorsysteme, TU Kaiserslautern, Kaiserslautern \\ Tel.: 0631-2053696, e-mail: freier@eit.uni-kl.de
}

\section{Kurzfassung}

Das vorgestellte Messprinzip für Kapazitäten wurde im Rahmen einer generischen Sensorschnittstelle entwickelt und basiert auf einem selbstkalibrierenden Schaltungskonzept mittels eines einfachen Operationsverstärkers. Es weist offsetarme sowie temperaturunempfindliche Eigenschaften auf und beinhaltet zwei Phasen: die erste Phase dient der Kalibrierung des Verstärkers, wobei das Messelement selbst verwendet wird, um die Offsetspannung auszulesen und zu speichern. In der zweiten Phase erfolgt die eigentliche Messung, in der gleichzeitig der Messverstärker durch das zu messende Sensorelement kompensiert wird. Auf einfache Weise sind somit Messgenauigkeiten von mindestens $2 \%$ bei einer Messrate von etwa $1 \mathrm{kHz}$ und 12 Bit Auflösung möglich. Der vorgesehene Messbereich erstreckt sich von $60 \mathrm{fF}$ bis $1,75 \mu \mathrm{F}$. Entwurf und Fertigung des Systems erfolgten in einer $0,35 \mu \mathrm{m}$ CMOS-Technologie, die Funktionalität wurde anhand eines Testchips und Musterkapazitäten im Bereich von 1,5 $\mathrm{nF}$ bis $33 \mathrm{nF}$ bestätigt.

\section{Einleitung}

Das hier präsentierte Messverfahren wurde unter der Zielsetzung einer möglichst großen Messbereichsabdeckung sowie der unkomplizierten und platzsparenden Realisierbarkeit zur Verwendung in einem Mixed-Signal-IC entworfen, welches als generische Schnittstelle zwischen Sensoren unterschiedlichen Typs 
und digitaler Signalverarbeitung dienen soll. Mittels dieses einzelnen ICs sind bis zu neun Messgrößen auslesbar, welche Widerstand, Kapazität, Induktivität, Spannung, Strom und Impedanz einschließen. Zweck ist der Aufbau von intelligenten Multi-Sensor-Systemen mit Fähigkeiten zur Selbstüberwachung, kalibrierung und -reparatur (z.B. [1]), wobei verschiedene Konzepte [2] [3] erstmalig zum Einsatz kommen. Die Funktionalität des eingesetzten und nachfolgend beschriebenen Verfahrens der Kapazitätsmessung beruht auf einem selbstkalibrierenden Schaltungskonzept, welches temperaturunabhängiges sowie drift- und offsetarmes Verhalten aufweist. Es wurde als Bestandteil des beschriebenen ICs implementiert, welcher in einer 0,35 $\mu \mathrm{m}$ CMOS-Technologie gefertigt wurde (Bild 1).

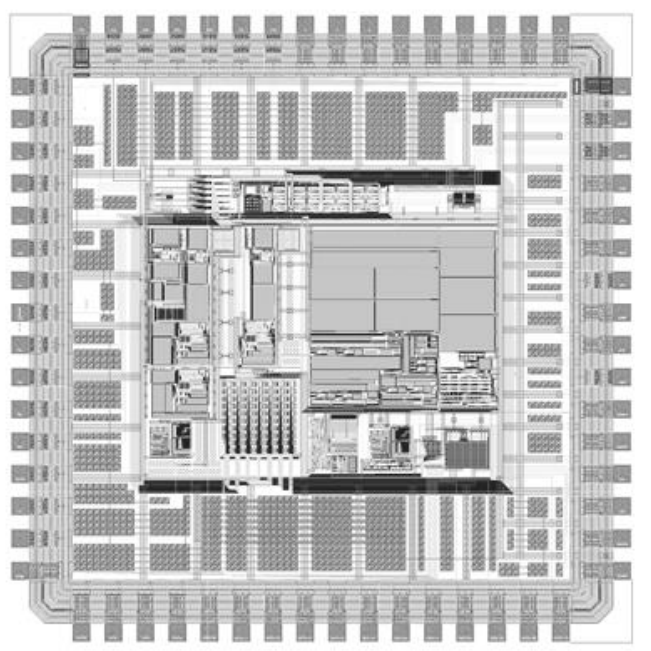

Bild 1: Chip-Layout der generischen Sensor-Schnittstelle in 0,35 $\mu \mathrm{m}$ CMOSTechnologie

Zum Stand der Technik existieren nur wenige integrierte Schnittstellen, die ein breiteres Spektrum an Sensoren aufnehmen können und dabei nicht auf einen einzelnen Typus beschränkt sind. Das einzige bekannte kommerziell verfügbare System, welches neben resistiven Größen zugleich auch kapazitive Sensoren auslesen kann, ist das „Universal Transducer Interface (UTI)“ der Firma Smartec [4]. Die Messung der angeschlossenen Sensorelemente erfolgt durch Anregung mit einer Wechselspannung, wobei der Ausgang ein periodenmoduliertes Signal ist. Zusätzlich zum eigentlichen Sensorelement wird noch ein weiteres Bauteil des gleichen Typs, kapazitiv oder resistiv, benötigt. Für kapazitive Wer- 
te liegt der maximale Messbereich zwischen 0 und 300pF. Neben diesem System gibt es noch eine Vielzahl an ICs, welche ausschließlich auf das Auslesen kapazitiver Sensoren festgelegt sind. Beispiele sind die ZMDI-Modelle ZSSC3122/3 [5] [6], welche mittels Charge-Balancing Größen bis zu 10pF (ZSSC3122) bzw. 260pF (ZSSC3123) messen können, oder die „PicoCap“ Serie von acam [7] [8], die Kapazitätswerte anhand einer Entladungszeitmessung bestimmt, wobei Messbereich und Genauigkeit von Referenzkondensatoren abhängen. Ein weiteres Verfahren wird u. a. im Analog Devices AD7745 [9] eingesetzt und als „Capacitance-to-Digital Converter“ bezeichnet, wobei mit einer effektiven Auflösung von 21 Bit mittels eines Sigma-Delta-Modulators die Ladung gemessen wird, welche sich durch Anregung mit einer Rechteckspannung auf dem Kondensator ansammelt.

\section{Prinzip der selbstkompensierenden Kapazitätsmessung}

Das vorgestellte Verfahren benötigt keine externen Bauelemente und basiert grundsätzlich auf einer herkömmlichen Methode, die zu messende Kapazität $C$ mittels einer Konstantstromquelle $I_{\text {ref }}$ bis auf einen bestimmten Spannungswert $U_{\text {ref }}$ zu laden und die dafür benötigte Zeit $t$ zu messen, woraus sich die Kapazität berechnen lässt:

$$
\begin{gathered}
C \cdot U_{r e f}=Q=I_{r e f} \cdot t \\
\Rightarrow \quad C=\frac{I_{r e f} \cdot t}{U_{r e f}}
\end{gathered}
$$

Hierzu stehen auf dem Chip bereits vorhandene Schaltungsblöcke zur Verfügung, welche nahezu temperaturunabhängige und einstellbare Referenzspannungen und -ströme generieren. Prinzipiell wird der Wert der zu messenden Kapazität als zeitliche Größe abgebildet. Mittels eines Komparators kann die Spannung am Kondensator mit der Referenzspannung $U_{\text {ref }}$ verglichen werden, wobei der Vergleich durch die Nichtidealität eines realen Komparators um dessen Offsetspannung verfälscht wird. Zur Kompensation dieses Fehlers kann die Messung in zwei Phasen aufgeteilt werden: 
Zunächst wird in der ersten Phase durch Nutzung der zu messenden Kapazität selbst das Offset des Komparators korrigiert, indem der Wert der Offsetspannung ausgelesen und auf der Kapazität gespeichert wird. Wie bei gängigen Auto-Zero-Verfahren wird der Komparator als Spannungsfolger konfiguriert und dessen Ausgang mit der Messkapazität verbunden. Der verbleibende Pol des Kondensators sowie der positive Eingang des Komparators werden auf Masse gelegt, sodass die Kapazität auf den Wert der Offsetspannung geladen wird (Bild 2). Zum Sicherstellen der Stabilität sollte hierfür aufgrund der Rückkopplung ein einfacher Operationsverstärker (OPV) als Komparator verwendet werden, an den allerdings keinen hohen Anforderungen bezüglich der Genauigkeit genügen gestellt werden, lediglich die Anstiegszeit sollte unterhalb der Periodendauer des verwendeten Taktsignals liegen.

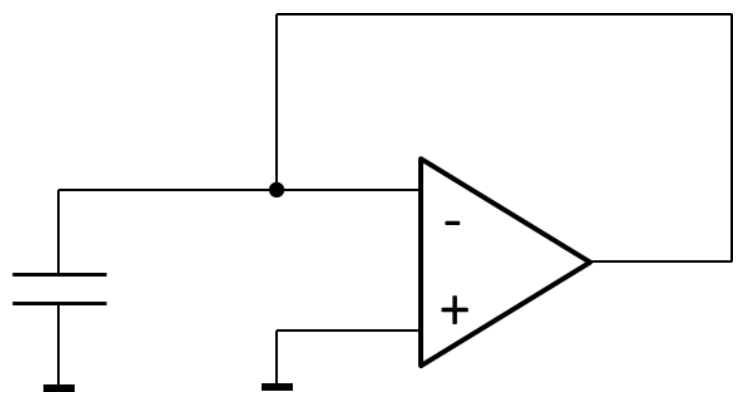

Bild 2: Phase 1 der Kapazitätsmessung umfasst das Auslesen und Speichern der Offsetspannung auf dem Messelement

In der zweiten Phase wird die eigentliche Messung durchgeführt, wobei der Offsetwert durch die Messkapazität selbst korrigiert wird. Wie in Bild 3 dargestellt, wird der Kontakt der Messkapazität, welcher zuvor mit dem Ausgang des Verstärkers verbunden war und noch immer an dessen negativen Eingang angeschlossen ist, zusätzlich mit einer Konstantstromquelle verbunden. Der andere, in der ersten Phase auf Masse liegende Kontakt, wird abhängig von der Richtung des Referenzstroms, entweder mit der positiven oder der negativen Versorgungsspannung verbunden und der positive Verstärkereingang auf eine Referenzspannung gelegt. Durch den Strom wird die mit der Offsetspannung vorgeladene Kapazität nun so lange weiter geladen, bis ihre Spannung der Referenzspannung plus Offset entspricht. Wird diese Spannung überschritten, schaltet der als Komparator verwendete OPV den Logikpegel an dessen Ausgang 
um. Dieses logische Signal dient als Ansteuerung der Zeitmessung zur Bestimmung der Dauer von Phase zwei, welche der Ladedauer vom Beginn bis zum Erreichen der Umschaltspannung entspricht. Die Zeitmessung geschieht hier anhand eines vorhandenen präzisen $4 \mathrm{MHz}$ Taktsignals sowie eines 12 Bit Zählers, welcher die Taktpulse bis zum Umschalten des Steuersignals registriert. Das Ergebnis der Messung liegt somit direkt als Digitalwert vor, was die weitere Verarbeitung erleichtert. Mit der gegebenen Taktfrequenz liegt die maximale Messdauer bei voller Ausnutzung der Zählergröße bei $1024 \mu$ s zuzüglich der Kompensationsphase von $0,5 \mu \mathrm{s}$.

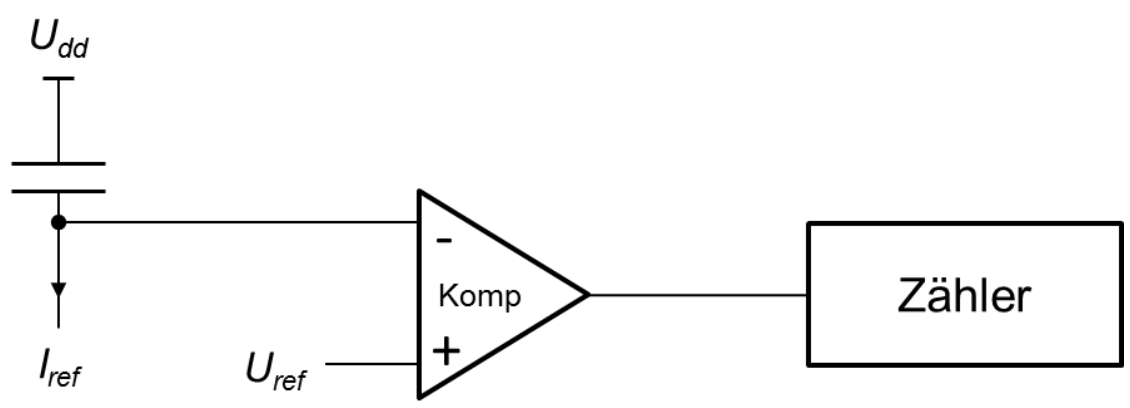

Bild 3: Phase 2 beinhaltet die kompensierte Kapazitätsmessung

Die Größen $U_{r e f}, I_{r e f}$ und die maximal bzw. minimal messbare Zeit legen den Messbereich des beschriebenen Verfahrens fest. Da die Kapazität mit der Betriebsspannung $U_{d d}$ verbunden wird, muss $U_{r e f}$ in Gleichung (2) durch $U_{d d}-U_{r e f}$ ersetzt werden. Auf dem beschriebenem IC sind die Werte von Strom und Spannung nominal einstellbar zwischen $0,5 \mu \mathrm{A}-2048 \mu \mathrm{A}$ und 1,22 V $-2,1 \mathrm{~V}$, woraus bei einer Betriebsspannung von $3,3 \mathrm{~V}$, einer Periodendauer des $4 \mathrm{MHz}$ Taktsignals von $250 \mathrm{~ns}$ und dem 12 Bit Zähler theoretisch messbare Werte zwischen ca. $60 \mathrm{fF}$ und 1,75 $\mu \mathrm{F}$ resultieren. Somit wird ein weiter Bereich sowohl integrierter als auch diskreter Kondensatoren abgedeckt. Da ebenfalls externe Referenzquellen verwendet werden können, lässt sich der Messbereich prinzipiell noch erweitern. Aufgrund der Temperaturkompensation der hier eingesetzten Referenzen ist die Kapazitätsmessung außerdem unempfindlich gegenüber Temperaturschwankungen. Weiterhin kann die parasitäre Kapazität der Messschaltung selbst ermittelt und kompensiert werden, indem zunächst eine Messung durchgeführt wird, noch bevor das eigentliche Messelement angeschlossen wird. 


\section{Realisierung und Ergebnisse}

Die schaltungstechnische Umsetzung des beschriebenen Messprinzips ist in Bild 4 dargestellt, die Implementierung erfolgte in der für das Gesamtsystem verwendeten 0,35 $\mu \mathrm{m}$ CMOS-Technologie.

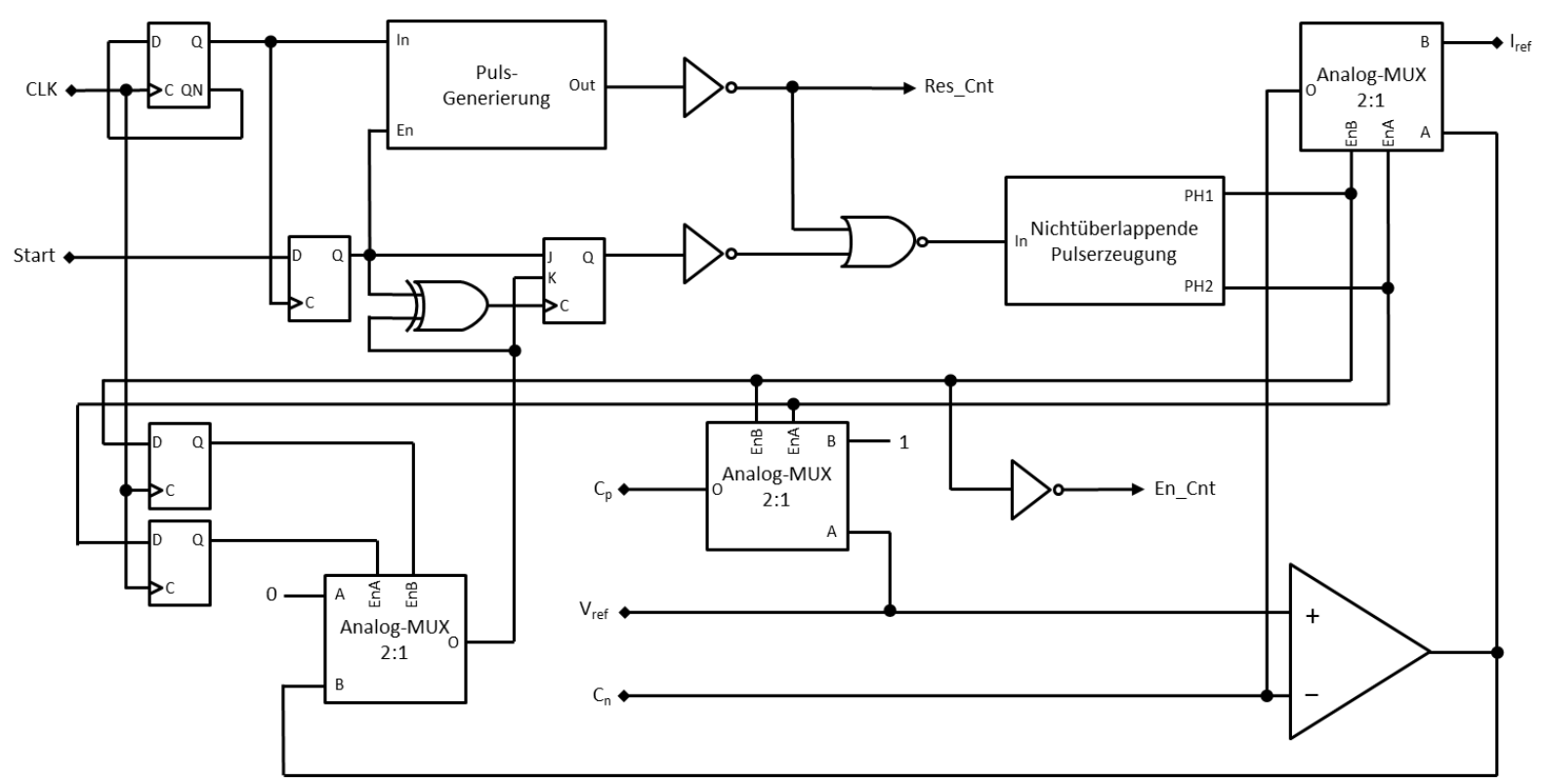

Bild 4: Umsetzung des Prinzips selbstkompensierenden Kapazitätsmessung auf Schaltplanebene

Tabelle 1 zeigt die Messergebnisse der hergestellten Schaltung an Kondensatoren, deren Bauteiltoleranzen jeweils mit $2 \%$ bzw. 5\% angegeben sind. Zum Eliminieren parasitärer Effekte wurde in den entsprechenden Messbereichen jeweils eine Messung ohne Kondensator durchgeführt und der ermittelte Zählerwert von den Messergebnissen abgezogen. Es fällt auf, dass die Resultate die grundsätzliche Funktionalität des Verfahrens bestätigen, sämtliche errechneten Kapazitätswerte jedoch kleiner sind als erwartet. Eine mögliche Erklärung hierfür können Abweichungen der Referenzgrößen von den zugrunde gelegten Werten sein, welche durch Messen ermittelt wurden. Kalibriert man die Kapazitätsmessungen, indem man eine konstante relative Abweichung im jeweiligen Messbereich unterstellt, so lässt sich anhand einer einzelnen Referenzmessung ein Korrekturfaktor für die weiteren Messungen im entsprechenden Bereich bestimmen. Der Korrekturfaktor ist dabei der Quotient aus Referenzwert und ge- 
messenem Wert. Die kalibrierten Messungen ergeben dann Werte, welche gänzlich innerhalb der Bauteiltoleranzen liegen (s. Tabelle 2). Da die wahren Bauteilwerte nicht bekannt sind, lässt dies nur den Rückschluss zu, dass die Ungenauigkeit des Verfahrens maximal der angegebenen Toleranz entspricht.

Tabelle 1: Messergebnisse an Testkondensatoren mit 2\% bzw. 5\% Toleranz

\begin{tabular}{|c|c|c|c|c|c|}
\hline Messbereich & $\boldsymbol{U}_{\boldsymbol{d} \boldsymbol{d}}-\boldsymbol{U}_{\boldsymbol{r e f}}$ & $\boldsymbol{I}_{\boldsymbol{r} \text { ef }}$ & Zählerwert & $\boldsymbol{C}$ berechnet & Bauteilwert \\
\hline $4,84 \mathrm{nF}$ & $1,672 \mathrm{~V}$ & $7,9 \mu \mathrm{A}$ & 2643 & $3,14 \mathrm{nF}$ & $3,3 \mathrm{nF}+/-2 \%$ \\
\hline $4,84 \mathrm{nF}$ & $1,672 \mathrm{~V}$ & $7,9 \mu \mathrm{A}$ & 1755 & $2,08 \mathrm{nF}$ & $2,2 \mathrm{nF}+/-2 \%$ \\
\hline $4,84 \mathrm{nF}$ & $1,672 \mathrm{~V}$ & $7,9 \mu \mathrm{A}$ & 1200 & $1,43 \mathrm{nF}$ & $1,5 \mathrm{nF}+/-2 \%$ \\
\hline $39,01 \mathrm{nF}$ & $1,672 \mathrm{~V}$ & $63,7 \mu \mathrm{A}$ & 2856 & $27,2 \mathrm{nF}$ & $33 \mathrm{nF}+/-5 \%$ \\
\hline $39,01 \mathrm{nF}$ & $1,672 \mathrm{~V}$ & $63,7 \mu \mathrm{A}$ & 1908 & $18,2 \mathrm{nF}$ & $22 \mathrm{nF}+/-5 \%$ \\
\hline $39,01 \mathrm{nF}$ & $1,672 \mathrm{~V}$ & $63,7 \mu \mathrm{A}$ & 1296 & $12,4 \mathrm{nF}$ & $15 \mathrm{nF}+/-5 \%$ \\
\hline
\end{tabular}

Tabelle 2: Kalibrierte Messergebnisse der Testkondensatoren

\begin{tabular}{|c|c|c|c|c|}
\hline Bauteilwert & $\boldsymbol{C}$ berechnet & Korrekturfaktor & $\boldsymbol{C}$ korrigiert & $\begin{array}{c}\text { Innerhalb } \\
\text { Bauteiltoleranz }\end{array}$ \\
\hline $3,3 \mathrm{nF}+/-2 \%$ & $3,14 \mathrm{nF}$ & $3,3 / 3,14$ & $3,3 \mathrm{nF}$ (Referenz) & - \\
\hline $2,2 \mathrm{nF}+/-2 \%$ & $2,08 \mathrm{nF}$ & $3,3 / 3,14$ & $2,19 \mathrm{nF}$ & $\checkmark$ \\
\hline $1,5 \mathrm{nF}+/-2 \%$ & $1,43 \mathrm{nF}$ & $3,3 / 3,14$ & $1,50 \mathrm{nF}$ & $\checkmark$ \\
\hline $33 \mathrm{nF}+/-5 \%$ & $27,2 \mathrm{nF}$ & $33 / 27,2$ & $33 \mathrm{nF}($ Referenz) & - \\
\hline $22 \mathrm{nF}+/-5 \%$ & $18,2 \mathrm{nF}$ & $33 / 27,2$ & $22,08 \mathrm{nF}$ & $\checkmark$ \\
\hline $15 \mathrm{nF}+/-5 \%$ & $12,4 \mathrm{nF}$ & $33 / 27,2$ & $15,04 \mathrm{nF}$ & $\checkmark$ \\
\hline
\end{tabular}

\section{Zusammenfassung}

Mit dem vorgestellten Verfahren wurde die Möglichkeit demonstriert, mit nur geringem Aufwand eine bereits vorhandene Schaltungsstruktur um die Fähigkeit einer relativ präzisen Kapazitätsmessung ohne externe Bauelemente zu erweitern, welche auch für vergleichsweise große Kapazitäten geeignet ist. Be- 
züglich der Genauigkeit von mindestens $2 \%$ kann das Verfahren zwar nicht mit beispielsweise dem des AD7745 konkurrieren, welcher eine absolute Genauigkeit von bis zu $4 \mathrm{fF}$ erreicht, allerdings ist die Messdauer mit etwa $1 \mathrm{~ms}$ bedeutend kürzer als die des AD7745, der pro Messung bis zu 100 ms beansprucht. Benötigt wird hauptsächlich ein einfacher Operationsverstärker, welcher in zwei Phasen durch die Nutzung des zu messenden Elements kompensiert wird. Die Ausgabe erfolgt in digitaler Form, ohne dass eine zusätzliche Wandlung des Signals notwendig ist. Bei Verwendung externer Referenzen kann außerdem der Messbereich nahezu beliebig erweitert werden.

\section{Literatur}

[1] M. A. Johar, R. Freier, A. König: Adding self-x capabilities to AMR sensors as a first step towards dependable embedded systems; Ninth Workshop on Intelligent Solutions in Embedded Systems (WISES), 41-46 (2011)

[2] R. Freier, S. Braun, A. König: Digital asynchronous on-chip temperature measurement using a buffer ring; 9th International Multi-Conference on Systems, Signals and Devices (SSD), 1-5 (2012)

[3] R. Freier, H. M. Adb, A. König: Adjustable phase-locked loop with independent frequency outputs; 10th IEEE International Conference on Semiconductor Electronics (ICSE), 517-521 (2012)

[4] Smartec BV: Universal Transducer Interface (UTI); (2010)

[5] Zentrum Mikroelektronik Dresden AG: ZSSC3122 cLite ${ }^{\mathrm{TM}}$ Low Voltage Capacitive Sensor Signal Conditioner; (2013)

[6] Zentrum Mikroelektronik Dresden AG: ZSSC3123 cLite ${ }^{\mathrm{TM}}$ Capacitive Sensor Signal Conditioner; (2012)

[7] acam-messelectronic gmbh: PcapØ1Ax-0301 Single-chip Solution for Capacitance Measurement; (2011)

[8] acam-messelectronic gmbh: PcapØ2A Single-chip Solution for Capacitance Measurement Volume 1: General Data and Front-End Description; (2013)

[9] Analog Devices, Inc.: AD7745/7746 24-Bit Capacitance-to-Digital Converter with Temperature Sensor; (2005) 